\title{
Entre el espejo, la máscara y la ausencia. Recorrido breve por la teoría de la autobiografía
}

\author{
GABRIELA KOESTINGER \\ Universidad Nacional Autónoma de México
}

\section{Resumen}

En tiempos recientes la escritura autobiográfica ha llegado a ocupar un lugar importante en la escena literaria; frente a esto, la teoría y la crítica han desarrollado una serie de herramientas conceptuales para dar sentido al fenómeno de las literaturas del yo. Este artículo presenta una revisión histórica de los principales conceptos que a partir del siglo XIX se desarrollaron en la teoría de la autobiografía. Se exploran las tres etapas propuestas por James Olney en la década de 1980; en primer lugar, la etapa del bios, compuesta por los trabajos de Wilhelm Dilthey y Georg Misch; en segundo lugar, se aborda la etapa del autos con los planteamientos de Georges Gusdorf y Philippe Lejeune; y finalmente se mira la etapa de la graphía o la ruptura postestructuralista con especial atención en el ensayo "Autobiografía como desfiguración" de Paul de Man.

Palabras clave: autobiografía; literaturas del yo; teoría de la autobiografía; sujeto; identidad

\begin{abstract}
In recent time, autobiographical writing has come to occupy an important place in the literary scene, in light of that, theoretical and critical writing have developed a set of conceptual tools to make sense of the phenomenon of self-writing. This essay presents a historical outline of the main concepts that were developed, since the 19th century, in Autobiographical Theory. It explores the three periods introduced by James Olney in the 80's; in first place the period of bios, composed by the works of Wilhelm Dilthey and Georg Misch; in second place there is the autos period with the proposals of Georges Gusdorf and Philippe Lejeune; and finally there is a look in the graphein period or the post-structuralist break with special attention to the essay "Autobiography as De-Facement" by Paul de Man.
\end{abstract}

Keywords: Autobiography; Self-Narratives; Autobiographical Theory; Self; Identity 
Sobre la evaporación y la centralización del Yo. Todo consiste en eso.

-Charles Baudelaire, Diarios intimos

\section{Introducción}

E n los últimos años la escritura autobiográfica ha adquirido una popularidad considerable, prueba de ello es que cada vez se publican más autobiografías, epistolarios, diarios íntimos, memorias o textos, como la autoficción, que transitan y juegan entre las fronteras de lo autobiográfico. Sin embargo, la autobiografía no es nueva en la historia de Occidente. Se puede hablar de escritos autobiográficos que datan de la Antigüedad, como la Carta VII de Platón, denominada por algunos pensadores como la "primera [auto] biografía" (García Fernández, 2007: 183); las Confesiones de San Agustín, del siglo IV; los Ensayos de Montaigne, del siglo XVII, o las Confesiones de Rousseau, del siglo XVIII. Si bien es complicado marcar un momento específico o un texto como el inicio de la autobiografía - ya que mucho depende del enfoque o la definición que se utilice-, lo que es certero es que la práctica de escribir sobre la vida de uno mismo ha estado presente desde tiempos ancestrales.

Curiosamente, las lecturas críticas sobre el género autobiográfico son bastante recientes. No es sino hasta finales del siglo XIX que se puede encontrar "the first wave of modern criticism on the field" (Smith y Watson, 2001: 113), y es en la segunda mitad del siglo XX donde tienen lugar las discusiones más ricas y fructíferas del campo. No obstante, el corto periodo en el que se ha desarrollado la teoría autobiográfica no ha impedido un extenso desarrollo, y su evolución a pasos agigantados ha situado la discusión en un lugar significativo en los estudios literarios: "As autobiography has been the dominant mode in literature of the twentieth century, so critical attention to the questions posed by the autobiographical act has become the principal preoccupation of theorists across the entire critical spectrum" (Olney, 1986: 621).

La teoría de la autobiografía cobra importancia en el contexto de la creciente producción de las literaturas del yo, pues contribuye a dar sentido no 
sólo a los textos y sus elementos o configuraciones, sino también a una serie de temas que se pueden vislumbrar en el circuito de lo autobiográfico, tales como sujeto, identidad, autor, escritura, intimidad, entre otros. Este texto, por tanto, pretende un breve recorrido histórico por la teoría de la autobiografía para comprender de dónde han partido y cómo se han desarrollado los principales conceptos que permiten interpretar, leer y acercarse de diferentes maneras al mundo de los géneros íntimos.

\section{Bios, autos, graphía}

Alrededor de la década de 1980, James Olney, uno de los teóricos más influyentes del campo en los últimos años, publicó una serie de ensayos sobre la teoría de la autobiografía. En textos como "Autos-Bios-Graphein: The Study of Autobiographical Literature" o "Autobiography and the Cultural Moment: A Thematic, Historical, and Bibliographical Introduction" estudió y organizó las diferentes lecturas críticas y teóricas que existían en torno a la escritura autobiográfica. Sus observaciones y propuestas se convirtieron rápidamente en un material fundamental para cualquier estudiante o investigador que se acerque al análisis de los textos autobiográficos.

Olney sugirió que se puede estudiar la teoría de la autobiografía en tres etapas históricas, que se ajustan a las tres raíces de la palabra autobiografía: autos, bios y graphía; a manera de encabezados, cada parte señala un acercamiento distinto al fenómeno autobiográfico. "What do we mean by the self, or himself (autos)? What do we mean by life (bios)? What significance do we impute to the act of writing (graphe) - what is the significance and the effect of transforming life, or a life, into a text?” (Olney, 1980: 6).

\section{Bios}

La primera etapa en la teoría de la autobiografía se caracteriza por el énfasis que se pone en la relación de los textos autobiográficos con la historia y en la importancia que se le da a la autobiografía como el retrato fiel de una vida. A 
finales del siglo XIX, Wilhelm Dilthey, filósofo e historiador alemán, concebía a la autobiografía como "the highest and most instructive form in which the understanding of life comes before us" (citado en Misch, 2002: 8), y abogaba por su uso en la escritura de la historia. Influenciado por Dilthey, el filólogo alemán Georg Misch continuó con el estudio sobre la autobiografía; para él, la cualidad autorreferencial de los textos autobiográficos producía un espacio único para el estudio de la vida humana, pues un escritor que habla sobre su propia vida es el que puede dar el más acertado y objetivo retrato de ésta: "the man who sets out to write the story of his own life has it in view as a whole, with unity and direction and a significance of its own" (Misch, 2002: 7).

Sin embargo, en esta perspectiva no se concibe la vida como una simple sucesión de acciones y procesos naturales, sino como una marcha acompañada de autoconciencia y conciencia sobre el mundo. Así que la autobiografía no sólo produciría conocimiento sobre eventos objetivos en una vida, sino que hablaría, también, de la vida tal como se experimentó, o como dice Misch (2002: 14): "life as actually lived".

En este sentido, la autobiografía produce conocimiento sobre la vida de un ser humano y sobre la manera en la que experimenta y reacciona al mundo, pero dicho conocimiento no se reduce a la vida de un individuo sino que, además, es el reflejo de una época: "Though essentially representations of individual personalities, autobiographies are bound always to be representative of their period" (Misch, 2002: 12). Así, Georg Misch comenzó a trabajar en una empresa ambiciosa: escribir la historia de la autobiografía desde la Antigüedad. Tal trabajo - esperaba Misch - mostraría "las formas peculiares en que el ser humano ordena su experiencia en un momento histórico determinado" (Loureiro, 1991: 2), y daría cuenta del proceso de conformación del hombre occidental.

Cabe destacar que para Misch las autobiografías de interés eran aquellas de los hombres ilustres que participaron en la vida pública de su época, aquellos que directamente provocaron cambios en la sociedad a través de puestos militares y políticos, como figuras intelectuales, etcétera, pues eran éstos los representativos de una sociedad en un periodo determinado (Smith y Watson, 2001: 114). El problema principal de este enfoque es que dejó fuera de consideración a otros tipos de escritura autobiográfica practicados por sujetos que no formaban 
parte de la esfera pública. Traigo esto a colación no sólo por lo interesantes que se presentan los estudios sobre autobiografía y su relación con conceptos como canon, poscolonialismo o feminismo, sino porque, aun cuando los criterios básicos de Misch fueron rápidamente superados, la actitud excluyente y jerarquizadora que plantea como legítima para una forma de escritura autobiográfica frente a otras, estableció la manera de acercarse a las autobiografías, así como de clasificarlas y valorarlas. Hasta hace relativamente poco tiempo, las autobiografías de sujetos más bien marginales permanecieron fuera de la circulación literaria o de la consideración en círculos académicos.

La historia de la autobiografía se vio inacabada por la muerte de Misch y el trabajo sólo llegó al Renacimiento, pero las aportaciones tanto de Wilhelm Dilthey como de Georg Misch fueron pasos indispensables para las consecuentes consideraciones teóricas sobre la autobiografía.

En términos generales, se puede decir que la etapa del Bios apela a una escritura que representa de manera transparente las ramas de la vida y de la conciencia humana, pues las autobiografías como conjunto forman un excelente medio para comprender el desarrollo histórico de la humanidad cuando se mira a los hombres que la han forjado. Enfocarse en el Bios implicó estudiar y valorar las autobiografías por el tipo de vidas que éstas retrataban así como por la información que proporcionaban a discursos como la psicología o la historia. En esta etapa, el trabajo del crítico es el de evaluar el carácter de quien escribe y la verdad histórica de los hechos que narra.

\section{Autos}

En la etapa del bios se elevó la autobiografía a un punto importante de atención crítica, sin embargo, el enfoque se quedó en la esfera del discurso histórico, pues la preocupación no era tanto por la autobiografía, como lo era por la historia y lo que ésta podía obtener de los textos autobiográficos. La segunda etapa, la del autos, se caracteriza por cuestionar la idea de textos transparentes y objetivos y concentrarse en el sujeto y su autorrepresentación. Ésta es la etapa considerada por diversos críticos como el inicio efectivo de una teoría de la autobiografía, pues se formularon las preguntas que al día de hoy siguen dirigiendo espacios 
importantes de la discusión y se dio "the application of a systematic literary analysis to canonical autobiography that parallels in intent and seriousness that addressed to the novel, poetry and drama" (Smith y Watson, 2001: 122).

James Olney atribuye el gran desplazamiento que inaugura la etapa del autos al teórico francés Georges Gusdorf con la publicación en 1956 del ensayo "Conditions et limites de l'autobiographie", texto seminal que problematizó más allá de lo que habían logrado sus antecesores cuando trasladó el enfoque de la relación entre autobiografía e historia hacia aquélla de autobiografía y sujeto (Loureiro, 1991: 2). Por otro lado, Sidonie Smith remarca la importancia del ensayo "Notes for an Anatomy of Modern Autobiography" de Francis R. Hart en el ámbito anglosajón, ya que el ensayo de Gusdorf impactó sobre todo en los círculos franceses (Smith y Watson, 2001 122).

De cualquier manera, se prefiera el trabajo de Gusdorf o el de Hart, los autores corresponden en las preocupaciones principales que caracterizan a la etapa del autos, ya que sus propuestas son parte, en un sentido más amplio, de cambios en la concepción general del sujeto. La idea de un sujeto unificado y universal se vio cuestionada por diversas reflexiones provenientes del marxismo, el psicoanálisis freudiano o la lingüística de Saussure (Smith y Watson, 2001: 123-124). Las nuevas corrientes apuntaban a un sujeto que perdía autoridad y agencia, un sujeto más bien interpelado por elementos como ideología, inconsciente o lenguaje. De ahí que, al pensar en autobiografía, la idea de un sujeto capaz de presentar su vida y su personalidad en un texto objetivo ya no era sostenible, "the project of self-representation could no longer be read as direct access to the truth of the self" (Smith y Watson, 2001: 125).

Gusdorf reconoce que el sujeto y la manera en la que se concibe ha fluctuado en el tiempo y en el espacio, y afirma, por tanto, que la autobiografía no ha existido siempre ni en todas las culturas. La autobiografía de la que habla Gusdorf es un producto exclusivamente occidental y de la tradición cristiana, de una conciencia de la vida individual separada de la colectiva y una concepción del tiempo como histórico en lugar de cíclico (Smith y Watson, 2001: 125).

Después de haber delimitado el espacio en el que se desarrolla la autobiografía, Gusdorf hace una distinción importante: si en la etapa del Bios el interés de la autobiografía se concentraba en las grandes vidas de los grandes hombres, 
es decir, en la esfera pública de la vida, para Gusdorf el valor de la escritura autorreferencial se encuentra en los recorridos que se hacen por la vida privada, aquel ámbito al que sólo puede acceder el hombre que habla de su propia vida, el historiador que se toma a sí mismo como objeto (Gusdorf, 1980: 31). Sin embargo - y es éste el gran aporte de Gusdorf-, la vida que retrata el escritor de una autobiografía no se dispone de manera exacta u objetiva, pues no es una imagen estática sino el retrato de un ser cruzado por el tiempo. La unidad presente en la autobiografía no viene de la vida misma, sino de un trabajo de reflexión posterior. El trabajo del autobiógrafo "is to reassemble the scattered elements of his individual life and to regroup them in a comprehensive sketch" (Gusdorf, 1980: 35), de manera que la condición de la autobiografía pasa de la objetividad a ser reconstrucción y ordenación.

Concebir la autobiografía como reconstrucción implica, entonces, hablar de interpretación o lectura, pues el ejercicio de escribirse (de manera retrospectiva) está influido por la memoria y por el hecho de que el sujeto que escribe desde el presente no es el mismo ya que fue en su pasado (Gusdorf, 1980: 39). En la autobiografía no sólo se encuentra una serie de hechos sino la toma de conciencia y la reflexión, por parte del escritor, de éstos; de ahí que Gusdorf hable de la autobiografía como una "segunda lectura de la experiencia" y aunque esto pudiera parecer un inconveniente, ya que la subjetividad amenazaría con alejar al sujeto de la máxima verdad a la que aspira, para Gusdorf la segunda lectura es más verdadera que la primera pues añade a la experiencia la conciencia sobre ésta (Gusdorf, 1980: 38). Una de las consecuencias del trabajo de interpretar y reordenar es atribuir nuevos significados a diferentes momentos de una vida; en la autobiografía el trabajo de ordenación implica algo más que la mera recapitulación de una vida, es el trabajo de un ser que busca y selecciona momentos específicos para decir quién es. Para justificarse y dar sentido a su persona, el sujeto autobiográfico se autodefine a través de lo que escribe: "[autobiography] does not show us the individual seen from outside in his visible actions but the person in his inner privacy, not as he was, not as he is, but as he believes and wishes himself to be and to have been" (Gusdorf, 1980: 45).

La última problemática a la que se acerca Gusdorf es la que se refiere a las dimensiones histórica, artística y antropológica de la autobiografía. Aunque no 
descarta la autobiografía como un documento útil para la historia, el teórico francés piensa que la dimensión artística es más importante, pues la armonía en el estilo o la belleza en las imágenes que articulan el recorrido hacia la verdad del hombre responden a una composición más bien literaria (Gusdorf, 1980 43). Sin embargo, para Gusdorf, el carácter artístico es secundario frente al antropológico, ya que la autobiografía sirve, en última instancia, para observar las maneras en las que el ser humano se conoce y se concibe.

Por tanto, Gusdorf apela por una crítica que se dé más allá del trabajo detectivesco de buscar y corregir inexactitudes históricas; él piensa en una crítica que pueda iluminar los significados más amplios en la escritura autobiográfica: "there is need of a second critique that instead of verifying the literal accuracy of the narrative or demonstrating its artistic value would attempt to draw out its innermost, private significance by viewing it as the symbol, as it were, or the parable of a consciousness in quest of its own truth" (Gusdorf, 1980: 44). Georges Gusdorf dedicó gran parte de su trabajo académico al estudio de las autobiografías; y aunque se pueden observar diferentes etapas y concepciones en su trayectoria, su proyecto general siempre se guió por un interés epistemológico en las formas del conocimiento y autoconocimiento humano (Camarero, 2008: 59).

Como se ha visto, la etapa del autos se inició gracias a una serie de dudas inquietantes frente al sujeto y su agencia; el sujeto y su autorrepresentación; o el sujeto y su relación con el pasado o la escritura, y todo desembocó en una idea de la autobiografía como producto de un ser dinámico que no sólo observa y habla de su vida, sino que crea los significados que la acompañan. En esta etapa la fórmula de Lequier "To create and in creating to be created" sería el lema perfecto para la autobiografía (Gusdorf, 1980: 44).

Ego es quien firma ego, o "El pacto autobiográfico"

Me parece necesario, antes de entrar a la etapa de la graphía, discutir el trabajo de Philippe Lejeune y particularmente su concepto del "pacto autobiográfico" (1975). Aun cuando James Olney omite la mención de Lejeune en la clasificación bios, autos, graphia -omisión injusta para algunos críticos como Paul John Eakin-, su trabajo es de una importancia capital, pues encendió el debate 
teórico sobre la autobiografía en Europa con sus análisis tan extensos como esquemáticos y minuciosos.

Es difícil situar a Lejeune en una de las categorías de Olney, lo cual no representa un problema mayor pues las categorías deben servir como una guía para entender el desarrollo teórico sobre la autobiografía y no como un índice restrictivo para hablar - o no hablar- de diferentes autores y conceptos. El trabajo de Lejeune es difícil de catalogar pues sus formulaciones no parecen encajar estrictamente en una etapa u otra, de ahí que me aventure al decir que Lejeune sirve como un puente entre la etapa del autos y la graphía ya que sus planteamientos parecen centrarse en una primera instancia en el sujeto, pero con la introducción de una discusión lingüística se alejan del autos, sin llegar a explotar plenamente las preocupaciones que se tratarían en la etapa de la graphía.

Lejeune, en su emblemático texto "El pacto autobiográfico" (1975), se esfuerza por encontrar las características particulares que separan a la autobiografía de otras escrituras autobiográficas posibles (memorias, epístola, novela autobiográfica, diario íntimo, etcétera) o géneros literarios; para esto brinda su famosa definición de autobiografía: "Relato retrospectivo en prosa que una persona real hace de su propia existencia, poniendo énfasis en su vida individual y, en particular, en la historia de su personalidad" (Lejeune, 1994: 50).

Con esta definición, Lejeune introduce parámetros tales como la forma del lenguaje o el manejo del tiempo, con los cuales separa la autobiografía de textos como el diario íntimo (que no cumple con la narración retrospectiva), las memorias o el poema autobiográfico. Sin embargo, el crítico francés encuentra que la definición no permite distinguir totalmente entre la autobiografía y la novela autobiográfica, por lo que el resto de su ensayo se guía por un esfuerzo de justificar teóricamente una diferencia entre el discurso ficticio de la novela autobiográfica y el factual de la autobiografía. Al notar que no hay características exclusivamente textuales que sirvan para dicha distinción, Lejeune tiene que recurrir a elementos extratextuales e introduce la equivalencia autor/narrador/ personaje como el elemento esencial para la existencia de la autobiografía.

Narrador y personaje son instancias textuales que, a través de pronombres, no remiten más que al sujeto de la enunciación, y como bien lo apunta Lejeune, 
siguiendo a Benveniste, "ningún pronombre personal, posesivo, demostrativo, etc., ha remitido jamás a un concepto, sino que ejerce simplemente una función que consiste en enviar a un nombre o a una entidad susceptible de ser designada por un nombre" (Lejeune, 1994: 59), de ahí que Lejeune encuentre la solución añadiendo al autor real en la ecuación. Para el teórico francés la marca del autor reside en el nombre propio, y es éste, como categoría léxica, el que funciona como marca textual de una entidad extratextual que sería la persona real: "una persona cuya existencia está atestiguada por su estado civil y es verificable" (Lejeune, 1994: 60).

Pero Lejeune lleva más lejos su conceptualización cuando decide situar la particularidad de la autobiografía desde la situación del lector e introduce así el denominado "pacto autobiográfico", un tipo de contrato social que existe entre el lector y el autor o la firma del autor. El pacto autobiográfico refiere una manera de acercarse a un texto y de leerlo, con la certeza absoluta de que lo que se lee pertenece al orden de lo real:

Cuando, para distinguir la ficción de la autobiografía, se trata de determinar a qué remite el yo de las narraciones personales, no hay necesidad de referirse a una imposible referencialidad extratextual: el mismo texto ofrece a fin de cuentas ese último término, el nombre propio del autor, a la vez textual e indudablemente referencial. Si esta referencia es indudable es por estar fundada en dos instituciones sociales: el estado civil [... ] y el contrato de publicación; no tenemos, por lo tanto, razón alguna para dudar de la identidad (Lejeune, 1994: 75).

Lejeune termina su ensayo argumentando que la autobiografía se define, entonces, por el tipo de lectura que provoca y llama a la autobiografía un género "contractual", para proponer que otros tipos de textos conllevan otros tipos de lectura y contrato, como la novela y el pacto novelesco. El texto concluye brindando una idea de cómo estudiar las autobiografías a través del tiempo: "La historia de la autobiografía sería entonces, más que nada, la de sus modos de lectura: historia comparada en la que se podría hacer dialogar a los contratos de lectura propuestos por diferentes textos [...] y los diferentes tipos de lecturas a que esos textos son sometidos" (Lejeune, 1994: 87). 
El trabajo de Lejeune no está exento de críticas y objeciones. El mismo Gusdorf en un congreso en la Sorbona criticó las propuestas de "El pacto autobiográfico" pues veía en ellas y en su espíritu de disección una serie de ataques arrogantes a la humanidad que realmente caracteriza a la autobiografía (Olney,1980: 18). Por otro lado, la famosa definición se ha enfrentado a innumerables objeciones. Para algunos críticos, afirmar que la autobiografía sólo puede estar escrita en prosa implica excluir a grandes nombres y trabajos como los de Byron o Whitman (el mismo Paul de Man articula su teoría de la autobiografía con la observación de un poema de Wordsworth). Por su parte, John Paul Eakin concuerda con Lejeune: "yo estaría de acuerdo en que la autobiografía tiende a darse en prosa, por la razón lógica de que la narrativa es la forma literaria temporal por excelencia" (Eakin, 1994: 12), aunque se puede notar la diferencia con el tono más bien excluyente de Lejeune.

Meri Torras, de manera sarcástica, cuestiona la delimitación temática que plantea la definición: “¿qué parte de la existencia cree Lejeune que debe llegar a contarse para que se trate efectivamente de una autobiografía? ¿En qué se mide? ¿En años? ¿En conflictos vitales? ¿En canas?” (Torras Francès, 1998: 14). Cree que la definición es, en general, prescriptiva y excluyente al privilegiar una forma de escritura autobiográfica frente a otras posibles: “¿por qué una de las 'formas íntimas de la literatura' se erige por encima del resto como muestra de la auténtica autobiografía y relega despóticamente las otras al reino de las no-autobiografías o de las autobiografías mutiladas?" (Torras Francès, 1998: 14).

Cabe destacar que el concepto de pacto autobiogáfico se desarrolló en una época en la que el estructuralismo ya había causado estragos; las certezas sobre la referencialidad del lenguaje o de la figura del autor como origen único del texto se encontraban desafiadas por la lingüística, la narratología o la teoría literaria en general. Los planteamientos y esquemas de Lejeune resonaron como prescriptivos en un contexto que buscaba ampliar los horizontes de lectura e interpretación de los textos, no reducirlos a contratos inviolables. Sin embargo, concuerdo con José María Pozuelo cuando dice: “Que el 'yo' autobiográfico sea un discurso ficcional, en los términos de su semántica, de su ser lenguaje construido, de tener que predicarse en el mismo lugar como otredad no impide que la autobiografía sea propuesta y pueda ser leída - y de hecho lo sea tantas 
veces así- como un discurso con atributos de verdad" (Pozuelo, 1993: 202). Es decir, se puede deconstruir y explorar el discurso autobiográfico para encontrar en el lenguaje una serie de imposibilidades como la de representación y referencialidad, pero eso no previene que la autobiografía se llegue a leer e interpretar como un documento factual. Como el mismo Lejeune lo marca, cuando en la lectura de una autobiografía surge una duda respecto de un evento retratado, la duda misma implica el valor de verdad que se le atribuye al texto, pues no se dudaría de un texto ficcional cuando el pacto implícito es uno que ronda más bien por los campos de la invención y lo imaginativo.

Pensar en el lector de las autobiografías se presenta entonces como un punto importante de discusión, quizá no en los términos jurídicos de Lejeune, pero importante a final de cuentas.

\section{Graphía}

Este desarrollo cada vez asemeja más a un camino de dudas que crecen de manera exponencial, y la etapa de la graphía no es una excepción, en todo caso es una explosión donde las certezas de la autobiografía (si aún existían) se deshacen en una serie de sospechas que cada vez abarcan más elementos. Si bien la característica principal de esta etapa es el giro hacia el lenguaje, es también la etapa que abarca o, en todo caso, abre un sinfín de horizontes para el estudio de la autobiografía. Influido por la ruptura postestructuralista en los estudios literarios, el campo de los géneros íntimos se nutre de sus ansiedades: se reta la idea de un sujeto anterior e inalterable que existe fuera del discurso; hay una percepción de los textos como inestables; la autorrepresentación se piensa más bien como dinámica y discursiva; se desconfía de la estabilidad de las categorías genéricas; y las discusiones sobre verdad y referencialidad divergen en mayor medida de las concepciones tempranas sobre autobiografía (Smith y Watson, 2001: 142-143).

Hoy en día, los estudios críticos sobre la autobiografía están en constante relación con campos como el de los estudios culturales, el feminismo, el poscolonialismo, etcétera, y pretender la mención de todas las diversas aproximaciones es ya una empresa utópica, por lo que me voy a concentrar, específicamente, 
en el texto de Paul de Man "Autobiography as De-facement" publicado en 1979, ya que proporciona una excelente herramienta para acercarse a la dimensión lingüística y retórica presente en los textos autobiográficos, o como menciona Loureiro "si algún valor tiene la aportación de De Man es hacernos perder definitivamente la inocencia (o la ceguera) con que nos hemos acercado hasta ahora a la autobiografía" (Loureiro, 1991: 6).

Paul de Man, teórico belga, considerado uno de los exponentes de la deconstrucción en Estados Unidos, empieza su texto criticando dos aspectos particulares que piensa han entorpecido los planteamientos de la teoría de la autobiografía. ${ }^{1}$ Por un lado, arremete en contra de la necesidad constante que ha tenido la crítica tradicional de definir genéricamente la autobiografía, pues la discusión le parece estéril al trabajar un conjunto de textos tan heterogéneos como los autobiogáficos; por otro lado, en el panorama postestructuralista, la teoría de los géneros es muy cuestionada y discutida, como marca Leigh Gilmore, "many postmodern explorations [...] seek to do without or do away with the definitional heft and limits provided by genre theory" (Gilmore, 1994: 6). En segundo lugar, De Man se acerca a la manera en que se ha intentado definir la autobiografía en contraposición a la ficción y observa cómo se ha asumido que la referencialidad, representación y diégesis son más simples y directas en la autobiografía, pero él (y es aquí donde introduce el primer punto que radicalizaría la discusión) reta dicha concepción:

are we so certain that autobiography depends on reference, as a photograph depends on its subject or a (realistic) picture on its model? We assume that life produces the autobiography as an act produces its consequences, but can we not suggest, with equal justice, that the autobiographical project may itself produce and determine the life and that whatever the writer does is in fact governed by the technical demands of self-portraiture and thus

1 Este punto se puede entender de manera más amplia con las problemáticas desarrolladas en el texto "The Law of Genre" de Jacques Derrida y, en general, las discusiones postestructuralistas que cuestionan la teoría de los géneros. 
determined, in all its aspects, by the resources of his medium? (De Man, 1979: 920).

El primer movimiento de De Man implica la propuesta de que aquella vida que leemos en una autobiografía tal vez no está determinada por la vida que efectivamente el autor vivió o no, sino por los requerimientos y las convenciones del discurso, y no se refiere a esto con una visión simplista donde el autor manipula la información, sino como una característica donde "the writing of autobiography will be interrupted and disrupted by the arbitrary effects of language" (McQuilan, 2001: 75). Una de las características primordiales en la etapa de la graphía, y una consecuencia muy presente en el ensayo de De Man, es la des-autorización del autor; pues así como Yeats llegó a decir "I have changed nothing to my knowledge and yet it must be that I have changed many things without my knowledge" en el prefacio a su autobiografía, el autobiógrafo visto en esta etapa pierde agencia, no es ya el productor consciente del sentido y es más una figura del discurso que su origen único.

Sugerir que las referencias del texto no son anteriores sino producidas por el discurso mismo presenta una situación en la que se vuelve imposible distinguir entre autobiografía o ficción, por lo que De Man propone la autobiografía no como un género sino como un modo de textualidad que puede estar presente en cualquier texto. Cuando en un texto el sujeto se presenta como el objeto de su propio entendimiento, entra en una estructura especular donde los dos yos implicados se determinan el uno al otro por relaciones de similitud y diferencia (De Man, 1979: 921); la estructura especular es la manifestación de una estructura lingüística y tropológica basada en la prosopopeya:

The fiction of an apostrophe to an absent, deceased or voiceless entity, which posits the possibility of the latter's reply and confers upon it the power of speech. Voice assumes mouth, eye and finally face, a chain that is manifest in the etymology of the trope's name prosopon poien, to confer a mask or a face (prosopon). Prosopopeia is the trope of autobiography, by which one's name [...] is made as intelligible and memorable as a face. Our topic deals with the giving and taking away of faces, with face and deface, figure, figuration and disfiguration (De Man, 1979: 926). 
Así, la autobiografía no sería la representación directa de una vida y de un sujeto, sino una construcción retórica, la ambición referencial se transforma en ilusión y el referente se disuelve en una ficción. En la estructura especular, la prosopopeya busca la figuración del sujeto, pero al estar el lenguaje conformado por tropos, al ser figuración "it is indeed not the thing itself but the representation, the picture of the thing" (De Man, 1979: 930); de ahí que la figuración lleve a una irremediable des-figuración de un sujeto que prueba ser imposible de aprehender. Por lo tanto, para De Man, el valor de la autobiografía no está en el conocimiento que produce sobre el sujeto - pues no lo produce- sino en que demuestra, a través de la estructura de sustitución, la imposibilidad de la totalización de todos los lenguajes (De Man, 1979: 922); es decir, la imposibilidad de aprehender completamente los objetos o sujetos que busca, "language both gives a voice and takes it away" (Anderson, 2001: 13).

No me parece muy difícil observar el gran salto que implica la conceptualización de De Man con respecto a críticos como Misch, Lejeune o Gusdorf; desde su perspectiva, tanto el sujeto como el texto están disueltos en el lenguaje, un lenguaje que ya no es una vía de acceso al sujeto y sus complejidades sino un fenómeno complejo en sí, una estructura que, con la retórica, construye y al mismo tiempo priva. "Autobiography as De-facement" representa un enfrentamiento radical con todas las ideas previas sobre el fenómeno autobiográfico, sus objetos o sujetos, y sus alcances o límites. De Man concluye, sin dejar de ser radical: "Death is a displaced name for a linguistic predicament, and the restoration of mortality by autobiography (the prosopopeia of the voice and the name) deprives and disfigures to the precise extent that it restores. Autobiography veils a defacement of the mind of which it is itself the cause" (De Man, 1979: 930).

\section{A manera de conclusión o sobre el boom en la teoría de la autobiografía}

Se puede preguntar por qué es tan reciente el interés teórico en lo autobiográfico y una posible explicación se podría encontrar en la posición que han ocupado los géneros íntimos en el espectro literario; el "continente oscuro de la literatura" - como ha llamado a la autobiografía Stephen A. Shapiro- ocupaba 
una posición inestable en la que el estatus literario o no de las escrituras íntimas se encontraba indeciso. La tradición teórica privilegió por mucho tiempo la idea de una literatura definida por sus valores ficcionales y estéticos (Shapiro, 1968: 423), y la autobiografía se había visto más bien ligada a discursos como el religioso, el histórico o incluso el psicológico.

La inclusión de los géneros íntimos en la circulación literaria es reciente, basta con observar los múltiples textos autobiográficos que ahora se incluyen en las obras completas de autores consolidados, la publicación en vida de diarios íntimos o la edición cada vez más frecuente de epistolarios. El interés de los estudiosos de la literatura en el fenómeno autobiográfico estuvo condicionado por una serie de movimientos tanto en el sistema literario como en el contexto intelectual, filosófico y social.

Por otro lado, y a pesar de lo reciente que es la teoría de la autobiografía, tambien se puede preguntar el porqué de su crecimiento acelerado; desde los trabajos de Gusdorf en la segunda mitad del siglo XX la discusión no sólo ha escalado rápidamente sino que se ha expandido de manera que hoy es tema importante de conversación en eventos y publicaciones internacionales. Cabe destacar, no obstante, que el surgimiento de este interés no es en lo absoluto espontáneo pues una serie de factores sociales y filosóficos le sirvieron como condiciones de posibilidad; como bien anota Sidonie Smith: la visión de un sujeto como universal y trascendental en la Ilustración; el individualismo radical impulsado por el romanticismo; el mito del self-made man de la Revolución Industrial; el paradigma del "gran hombre" en el discurso histórico; o el psicoanálisis freudiano y sus herramientas para la autorreflexión (Smith y Watson, 2001: 112) crearon un terreno fecundo para el interés creciente tanto en la escritura autobiográfica como en sus lecturas críticas.

Por su parte, James Olney piensa que la inclusión de la autobiografía en las discusiones literarias tuvo que ver con el giro que se dio del bios al autos, es decir, cuando las preocupaciones pasaron de la vida al sujeto en sí, ya que "behind every work of literature there is an 'I' informing the whole and making its presence felt at every critical point, and without this 'I', stated or implied, the work would collapse into mere insignificance" (Olney, 1980: 22). Por otro lado, reflexiona sobre el crecimiento apresurado y, al observar la gran variedad de 
aproximaciones, concluye que, a pesar de las diferencias, todas las reflexiones vienen de una ansiedad por el sujeto y la autoconciencia y el autoconocimiento: "the heart of the explanation for the special appeal of autobiography to students of literature in recent times: it is a fascination with the self and its profound, its endless mysteries and, accompanying that fascination, an anxiety about the self, an anxiety about the dimness and vulnerability of that entity that no one has ever seen or touched or tasted" (Olney, 1980: 23).

Si bien el enfoque principal en la etapa del bios era histórico, su búsqueda no dejó de ser una incesante preocupación por la conformación del hombre; la etapa del autos transformó el paradigma y convirtió el terreno autobiográfico en uno fecundo para las reflexiones sobre el sujeto, pues vio en la autobiografía el camino ideal para el autoconocimiento; y la etapa de la graphía o de la ruptura postestructuralista, aun cuando explotó todas las ansiedades, aun cuando está más influida por imposibilidades que por certezas, no deja de agonizar por la idea de ese sujeto que busca negar: "however much they talk about genre or linguistics or deep-lying structures, what they are still troubling about is the self and consciousness or knowledge of it, even though in a kind of a bravura way some of them may be denying rather than affirming its reality or its possibility" (Olney, 1980: 23).

Las diferentes reflexiones teóricas sobre la autobiografía se han desarrollado en relación con las valoraciones y concepciones generales sobre temas como el sujeto, la individualidad, la literatura, la escritura o el lenguaje. Abordar el desarrollo histórico de la teoría autobiográfica permite destacar todas las complejidades de un objeto que tal vez, en primera instancia, se podría pensar como simple y transparente. Por otro lado, tanto la clasificación de James Olney como las aportaciones de los críticos mencionados sirven como una primera herramienta para acercarse de una manera menos inocente al fenómeno de las literaturas del yo.

\section{Referencias bibliográficas}

ANDERSON, Linda (2001): Autobiography, Londres: Routledge. 
CAmarero, Jesús (2008): "La théorie de l'Autobiographie de Georges Gusdorf". En Çedille. Revista de Estudios Franceses. Núm. 4, pp. 57-82.

De Man, Paul (1979): “Autobiography as De-facement”. En MLN. Vol. 94, núm. 5 (Comparative Literature), pp. 919-930.

EAKIN, Paul John (1994): “Introducción”. En Philippe Lejeune, El pacto autobiográfico y otros estudios. Madrid: Megazul / Endymion, pp. 9-46.

García Fernández, Román (2007): “La Carta VII. La autobiografía de Platón y su método”. En Eikasia. Revista de Filosofía. Núm. 12, pp. 163-183.

GILMORE, Leigh (1994): “The Mark of Autobiography: Postmodernism, Autobiography, and Genre". En Kathleen Ashley, Leigh Gilmore y Gerald Peters (eds.), Autobiography and Postmodernism. Boston: The University of Massachusetts Press, pp. 3-18.

Gusdorf, Georges (1980): “Conditions and Limits of Autobiography”. En James Olney (ed.), Autobiography: Essays Theoretical and Critical. Nueva Jersey: Princeton University Press, pp. 28-48.

Leleune, Philippe (1994): El pacto autobiográfico y otros estudios. Madrid: Megazul / Endymion.

Loureiro, Ángel (1991): "Problemas teóricos de la autobiografía”. En Ángel Loureiro (coord.), La autobiografía y sus problemas teóricos. Estudios e investigación documental. Barcelona: Anthropos, pp. 2-8 (Monografías temáticas, 29).

McQuilan, Martin (2001): Paul de Man. Londres: Routledge.

Misch, Georg (2002): A History of Autobiography in Antiquity. Londres: Routledge.

Olney, James (1980): "Autobiography and the Cultural Moment: A Thematic, Historical, and Bibliographical Introduction". En Olney, James, Autobiography: Essays Theoretical and Critical. Nueva Jersey: Princeton University Press, 1980, pp. 3-27. 
OlneY, James. (1986): "Rev. of Fictions in Autobiography, Paul John Eakin”. En American Literature. Núm. 58, pp. 621-623.

Pozuelo Yvancos, José María (1993): "La frontera autobiográfica”. En Poética de la ficción. Madrid: Síntesis, pp. 179-225.

Shapiro, Stephen A. (1968): "The Dark Continent of Literature: Autobiography”. En Comparative Literature Studies. Vol. 5, núm. 4, pp. 421-454.

Smith, Sidonie; y Watson, Julia (2001): Reading Autobiography. A Guide for Interpreting Life Narratives. Minneapolis: University of Minnesota Press.

TORRAS FranCÈs, Meri (1998): La epístola privada como género: estrategias de construcción [tesis doctoral]. Barcelona: Bellaterra / Universidad Autónoma de Barcelona. 